TI 2013-084/ VIII

Tinbergen Institute Discussion Paper

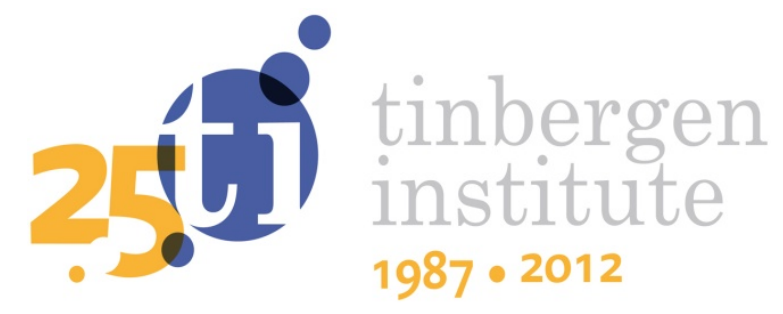

Towards a General Theory of Mixed

Zones:

The Role of Congestion

\author{
Yuval Kantor \\ Piet Rietveld \\ Jos van Ommeren
}

Faculty of Economics and Business Administration, VU University Amsterdam, and Tinbergen Institute. 
Tinbergen Institute is the graduate school and research institute in economics of Erasmus University Rotterdam, the University of Amsterdam and VU University Amsterdam.

More TI discussion papers can be downloaded at http://www.tinbergen.nl

Tinbergen Institute has two locations:

Tinbergen Institute Amsterdam

Gustav Mahlerplein 117

1082 MS Amsterdam

The Netherlands

Tel.: +31(0)205251600

Tinbergen Institute Rotterdam

Burg. Oudlaan 50

3062 PA Rotterdam

The Netherlands

Tel.: +31(0)10 4088900

Fax: $+31(0) 104089031$

Duisenberg school of finance is a collaboration of the Dutch financial sector and universities, with the ambition to support innovative research and offer top quality academic education in core areas of finance.

DSF research papers can be downloaded at: http://www.dsf.nl/

Duisenberg school of finance

Gustav Mahlerplein 117

1082 MS Amsterdam

The Netherlands

Tel.: +31(0)20 5258579 


\title{
Towards a General Theory of Mixed Zones: The Role of Congestion
}

\author{
Yuval Kantor \\ Piet Rietveld \\ Jos van Ommeren \\ Department of Spatial Economics, VU University Amsterdam
}

25 April 2013

\begin{abstract}
Mixed commercial and residential land use is observed in most cities around the world. This is in contrast to a myriad of bid rent models, which predict that mixed land use does not occur. The main exception are the models by Fujita and Ogawa (1982) and Lucas and Rossi-Hansberg (2002) that predict the presence of a very restrictive type of mixed land use. The latter study derives the equilibrium distribution of residential and commercial land uses while allowing for endogenous agglomeration externalities. We extend this model by introducing a traffic congestion externality. We show that congestion induces a general type of mixed land use zone, which is comparable to the type of zone assumed in the model of Wheaton (2004). The interplay between these externalities is then demonstrated, as reduced congestion leads to commercial concentration and agglomeration gains.
\end{abstract}

This paper was written in the context of NWO Sustainable Accessibility for the Randstad (DBR) program. We would like to thank the participants of Tinbergen Institute lunch seminar, Rotterdam 2012, Kuhmo-Nectar, Berlin 2012, and the North American Regional Science Council conference, Ottawa 2012, for their comments. Van Ommeren and Rietveld are fellows of the Tinbergen Institute Amsterdam. Yuval Kantor is a student of the Tinbergen Institute Amsterdam. Corresponding author: Yuval Kantor (e-mail: y.kantor@vu.nl). 


\section{Introduction}

Over the last decades, a myriad of urban economic models have been developed which imply that residents and firms never mix within cities. These models assume that all characteristics of locations and residents are observed. Hence, residential and commercial land use are strictly separate. This is in contrast to the well-known observation that residential and commercial land use is almost always mixed in some parts of the city (Koster and Rouwendal, 2013). Because the locations of residences and firms define the commuting flows within cities, there is nowadays a large literature which discusses whether urban economic models can say anything useful about within-city commuting flows as observed in reality (for instance, Hamilton and Röell, 1982, and Giuliano and Small, 1993).

Importantly, our work relates to two seminal studies that have been able to show that under certain conditions mixed zones may emerge, but only of a special type (Fujita and Ogawa 1982; Lucas and Rossi-Hansberg, 2002, hereafter LRH). This type of mixed zone may be labeled balanced mixed zone. In balanced mixed zones, the number of residents and number of jobs are exactly equal to each other. Furthermore, all residents within a balanced mixed zone work in this zone. As a result, all residents in these mixed results do not commute, and there are no workers who live outside the mixed zone who commute through this zone (essentially a backyard economy). Although both papers have substantially improved our understanding of within-city agglomeration, we believe that it is fair to say that we currently have no general theory of mixed zones.

The observation that most land is in mixed use, motivated Wheaton (2004) to introduce an urban economic model which assumes mixed zones (for example, it may occur that at certain locations, there are 80 residents and 20 jobs). Similarly, Anas and Kim (1996) assume unobserved features of locations and random variations in utility, and show, as one may expect, that land use is mixed. The current paper aims to explore the possibility to derive mixed land use without introducing random elements.

In essence, we follow LRH who study the equilibrium distribution of residential and commercial land uses. Importantly, assuming a symmetric circular city, they allow for employment agglomeration externalities in line with a large empirical literature which shows that productivity of firms depends positively on nearby employment (Rosenthal and Strange, 2003). Our main 
contribution is that we introduce a traffic congestion externality to the model (see also Arnott, 2007, who combines both externalities in a setting of two connected islands). We demonstrate that the presence of this externality results in a general type of mixed residence-business land use zone, in which the number of workers does not equal the number of jobs. We label these areas unbalanced mixed zones.

To improve the intuition, why congestion results in the existence of unbalanced mixed zones in equilibrium, the following example is useful. Consider a balanced mixed use zone where bid rents of firms and residents coincide. Outside the zone, there are firms that are willing to pay a higher wage that makes commuting from the mixed zone more attractive than working locally. That, in turn, increases the bid rent of residents in the zone, and housing thus replaces businesses. In a model without congestion effects, the zone becomes purely residential. However, in our model the influx of workers and the increase of commuting give rise to congestion which entails higher travel costs and hence a lower net wage. If these costs become high enough, the workers' bid rent may decrease to coincide again with the firms' bid rent. In this new equilibrium, made possible by congestion, the zone is taken by both residents and firms, and some of the workers commute to jobs outside the zone.

To understand our contribution, we emphasize that the monocentric model is the basis of an extensive economic literature that has explored city spatial structure. This model studies a city consisting of a residential ring around a center, in which all jobs are concentrated (Mills, 1972). In the model's simplest form, city residents balance their desire to lower their commuting costs with their preference for spacious housing. The picture that emerges is that of a city in equilibrium, with residential densities and land rents decreasing with distance from the center. A great number of phenomena observed in real-world cities can be explained by this highly stylized model, from the setting of an urban boundary, through racial segregation, to commuters' choice of transport modes.

A drawback of this model is its assumption that firms' land use structure is fixed. By assuming a single central business district, other forms that emerge in reality are ruled out. However, urban regions often exhibit complex spatial patterns of residential and commercial organization (Anas et al., 1998). In the monocentric model, the structure is exogenously given, because it does not consider economic forces that shape the distribution of firms over the city. As a result, it excludes the 
possibility that firms and residents mix within zones, and thus implies single-use zones. However, as shown by Wheaton (2004), firms and residences are interspersed, and jobs are only slightly more centralized than houses. We develop an urban model in which the resulting zones may be single-use or mixed and have any composition of residences and firms: from balanced mixed zones in which people work where they live (also known as backyard economy) and where no commuting takes place, through unbalanced mixed zones which are dominated by one of the uses, to the classical single-use zones (labeled hereafter pure).

One of the strongest city-shaping forces is agglomeration externalities, which have attracted plenty of attention in urban economics. Proximity of firms to each other entails benefits such as exchanges of knowledge and ideas and sharing of labor pools and intermediary suppliers. By locating close to each other, firms enjoy higher productivity levels and lower costs. These advantages draw firms to cluster and lead to the growth of cities and commercial centers (Glaeser et al., 1992). Fujita and Ogawa (1982) specified the productivity advantages of agglomeration in a highly simplified form, and constructed a model where locations of both residents and firms are endogenously determined along a line segment representing a city. Under certain sets of parameters, equilibrium city structures of various sorts may emerge, including, but not limited to, the monocentric and duocentric forms, as well as a city composed of a single mixed zone of businesses and residents.

This model was extended by LRH, who relaxed many of its restrictive assumptions. Most notably, the assumed symmetrical equilibrium city structure was changed from linear to circular. LRH proved the existence of an equilibrium, and developed an algorithm for its construction. As in Fujita and Ogawa, different sets of underlying parameters give rise to cities of various types. The resulting cities all consist of a combination of zones that are either pure business zones, pure residential zones, or fully balanced mixed zones. We use their model as a springboard for further exploration of the urban structure.

Locating close to firms is advantageous for residents and other firms alike. The former enjoy lower commuting distances, while the latter benefit from agglomeration externalities. In many cities, however, locations with high density of population or economic activity suffer from negative congestion externalities (Arnott, 2007). It is then reasonable to assume that firms may opt for more 
remote locations, if city center congestion becomes a serious problem for their employees. On the other hand, residents may respond to congestion by moving towards central locations, leading to an increase in densities and rents in these locations. Congestion externalities are different in nature from agglomeration externalities, and both have important implications for urban structure. Rossi-Hansberg (2004) emphasized the relevance of road congestion, but assumed it away for convenience. We show that congestion is not simply the inverse force of agglomeration externalities (though it does produce more dispersed business activities than would otherwise arise). The presence of congestion enables the model to produce a diverse array of mixed land use patterns, which cannot be attained from the original LRH model. The interplay between employment and traffic congestion externalities is further demonstrated, as a reduction in congestion externalities leads to employment concentration and therefore agglomeration gains, whereas an increase in these externalities leads to labor dispersion and agglomeration losses.

The model developed in this paper can also be viewed as an extension to the one developed in Wheaton (2004), where households aim to minimize transportations costs, and firms aim to lower the wages they pay to workers. Wheaton shows that congestion leads to employment decentralization (in line with our findings). Nevertheless, two major differences between Wheaton's model and the one presented here are noteworthy. First, the land in Wheaton's market equilibrium is not occupied by the highest bidder, as is commonly assumed in the literature, but is rather shared between uses in proportion to their land bid-rent. Therefore, the model essentially assumes mixed land uses. In the current paper, we achieve mixed land use composition based on the common assumption of bid-rent competition. Second, spatial agglomeration advantages are exogenous to Wheaton's model, whereas in our model they are determined simultaneously with urban land use ${ }^{1}$.

The paper is structured as follows. Section 2 describes the model, focusing on extensions to LRH. Section 3 describes the algorithm for the construction of an equilibrium, and discusses the conditions under which mixed zones arise. The algorithm description is given special attention, since adding congestion in the model leads to substantial algorithmic challenges. Section 4 presents

\footnotetext{
${ }^{1}$ Wheaton's suggestions for extensions are implemented in our model, including two-way commuting, and endogenous land consumption by firms and households.
} 
numerical simulations of the model, resulting in various city structures. Section 5 concludes with a discussion of the model's implications.

\section{Model}

Our model follows much of the path marked by LRH (see LRH for a comprehensive description). We consider an open circular city of radius $S$ in which land is owned by absentee landlords. Locations are defined by their coordinates $(r, \phi)$ in a polar coordinate system with the city geographic center as its origin. The set of equilibria is restricted to symmetric cases, so a location can be denoted by $r$ alone. At each location $r$ within the city limit $(r<S)$, a share $\theta(r)$ of land is used by production activity, and the remaining $1-\theta(r)$ is for residential use. We denote labor density - employment per unit of production land - at location $r$ by $n(r) . N(r)$ denotes the number of people housed at $r$, per unit of residential land.

\section{Economic agents}

Firms produce a single good using land and labor ${ }^{2}$. Production at $r$, denoted as $x(r)$, is subject to a productivity externality of neighboring firms, denoted by $z(r)$. Production is assumed to be a constant returns to scale function of labor and land at $r$, and is expressed as $x(r)=g(z(r)) f(n(r))$.

Production at location $r$ is determined by labor density at all other locations $(s, \phi)$. We assume that the externality $z(r)$ takes a specific form, and declines exponentially at a rate $\delta$. Hence,

$$
z(r)=\int_{0}^{S} \int_{0}^{2 \pi} \delta e^{-\delta \Delta(r, s, \phi)} d \phi s \theta(s) n(s) d s,
$$

where $\Delta(r, s, \phi)$ denotes the distance between $(r, 0)$ and $(s, \phi)$. With this formulation, $\delta$ captures the locality of agglomeration effects, while it does not affect the average external effect.

\footnotetext{
${ }^{2}$ This good is traded with the larger economy.
} 
Workers enjoy consumption of the good $c$, and residential land $l$. Their utility function is denoted by $U(c, l)$, and is strictly increasing in $c$ and $l$. Workers are assumed to be fully mobile within and out of the city, so their utility level is equal at all locations:

$$
U(c(r), l(r))=\bar{u},
$$

where $\bar{u}$ is the exogenously given utility level prevailing in the larger economy. Each worker is endowed with one unit of labor time, which he supplies to production time and commuting time. For production, the worker receives an hourly wage $w$. When used for commuting, production time declines proportionally by a factor of $\tau$ per unit of distance traveled.

A key condition of LRH is that the total quantity of labor invested in production and commuting does not exceed the total labor supplied by workers in the city. Let $H(r)$ indicate the stock of workers in locations $s \in[0, r)$ that are unhoused in $[0, r)$. This variable is negative if there are more houses in these locations than labor used in production and commuting. Determining the value of $H(r)$ is an essential step in finding an equilibrium. In every small interval $[r, r+d r)$, the stock $H(r)$ is increased (decreased) by the excess (shortage) of people employed as compared to the people housed in this interval:

$$
2 \pi r[\theta(r) n(r)-(1-\theta(r)) N(r)] d r
$$

A positive value of $H(r)$ implies that $H(r)$ labor units commute from locations $s$, where $s>r$, towards the center ${ }^{3}$. To bring $H(r)$ units of labor from $r+d r$ to $r$ requires that $H(r)+H(r) \tau(r) d r$ labor units be brought to $r+d r$. The term $H(r) \tau(r) d r$ is the total time lost in commuting between $r+d r$ and $r$. A negative value of $H(r)$ implies an excess of residents in $[0, r)$, who need to be

\footnotetext{
${ }^{3}$ For example, one labor unit may be supplied by a single worker who does not commute. When commuting takes place, production time is reduced. One labor unit may also be supplied by two workers who lose half of their labor time on commuting.
} 
employed at locations $s \geq r$, and therefore commute away from the center. The total commuting costs over $d r$ are $-H(r) \tau(r) d r . H(r)$ is then defined by the differential equation:

$$
\frac{d H(r)}{d r}=2 \pi r[\theta(r) n(r)-(1-\theta(r)) N(r)]+|\tau(r) H(r)|,
$$

with the condition $H(0)=0$.

We now turn to determine the level of rent firms and residents are willing to pay. To derive the land use structure, we assume that land is allocated to the highest bidder. The bid is subject to the wage prevailing at each location, denoted by $w(r)$. The interpretation of $w(r)$ is determined by land use at $r$. At business locations, it denotes the wage per labor unit paid by local firms. At residential locations, it denotes the earnings net of travel costs, received by workers locally residing. The business bid rent $q(r ; w, z)$ is the highest bid a firm can make for land, which corresponds to the firm's profits per unit of land, defined as:

$$
q(r)=\max _{n}\{g(z(r)) f(n)-w(r) n\} .
$$

This equation determines the optimal labor input $n(r ; w, z)$, given the productivity and wages prevailing at each location.

The bid rent $Q(r)$ of residents is expressed as the highest rent level a resident at $r$ is willing to pay:

$$
Q(r)=\max _{c, l}\left\{\frac{w(r)-c}{l}\right\} \text { s.t. } u(c, l) \geq \bar{u}
$$

This equation determines the chosen land consumption, as well as its inverse, the residential density $N(r ; w)$. 
Transportation costs, congestion and wages

Commuting costs take the form of a loss of labor time that depends on the distance traveled to and from work each day, and on the congestion level along the way. Travelling through a small road segment, a worker loses a share of his remaining labor time. Denoting the labor supply remaining available at $r$ by $L(r)$, the rate of labor decline of workers traveling through location $r$ is defined by:

$$
\frac{d L}{d r}=-\tau(r) L(r)
$$

In LRH, $\tau(r)$ was assumed to be a constant, so $\tau=k$, where $k$ captures the time losses due to travel distance. We assume that $\tau(r)$ depends on $H(r)$, which captures the quantity of labor traveling at $r$ from the center, so $\frac{1}{2 \pi r}|H(r)|$ defines traffic density at $r$. We assume a generalized Solow(1973) congestion function:

$$
\tau(r)=\left\{\begin{array}{cc}
\kappa+\mathrm{t}\left(\frac{H(r)}{2 \pi \mathrm{r}}\right)^{\mathrm{T}} & \text { if } H>0 \\
-\kappa-\mathrm{t}\left(\frac{-H(r)}{2 \pi \mathrm{r}}\right)^{\mathrm{T}} & \text { if } H<0
\end{array},\right.
$$

where , $T \geq 0{ }^{4}$. The differential equation (6) implies a labor supply function. A worker who lives at $S$ and works at $r(r>s)$ supplies $L(r, s)=e^{-\int_{s}^{r} \tau(\rho) d \rho}$ hours of labor to the firm.

We assume that both labor mobility and firms' choice of labor are free, so in equilibrium no worker can gain by changing job location and firms cannot gain by changing the location from which they hire labor. These assumptions imply that workers who live in $r \in\left(r_{1}, r_{2}\right)$, which is a region in which all commuting is pointed to the same direction, receive a wage given by:

$$
w(r)=w\left(r_{1}\right) e^{-\int_{r_{1}}^{r} \tau(\rho) d \rho},
$$

\footnotetext{
${ }^{4}$ Solow (1973) assumes that $T=1$.
} 
so $\frac{d w(r)}{d r}=-\tau(r) w(r)$. Hence, the wage function depends on the level of congestion.

If no commuting takes place in the interval $\left(r_{1}, r_{2}\right)$, then this interval can only be a balanced mixed zone where workers are employed at their place of residence. Within this zone, residential and business bid rents are necessarily equal to each other. Therefore, the wage level at the mixed zone is the solution to:

$$
Q(w(r))=q(w(r) ; z(r)) .
$$

We call the set of wages that enable a mixed zone of any type the mixed wage curve, and denote it by $w_{m}(r)$.

\section{Solving the model using a two-step procedure}

LRH develop a novel, non-standard, algorithm to solve the model, which we take as the basis for our search for equilibrium. This algorithm consists of two steps, repeated iteratively until convergence. The first step computes equilibrium land use distribution, and therefore city structure, given $z(r)$. In the second step, a new productivity function $\tilde{z}(r)$ is obtained using equation (1), given the land use distribution obtained from the first step. One of the contributions of the current paper is to make assumptions about the travel congestion function, such that the model can be solved by adapting only the first step $^{5}$. There is no need to introduce another step, where congestion levels are calculated. Because the stock of unhoused workers $H(r)$ is used as a measure of road usage, congestion levels, $H(r)$ and $w(r)$, are simultaneously computed in the first step. This step is now described in detail.

To find an equilibrium structure, one must search for a consistent land use pattern, in which all workers are housed in the city, and all residents are employed, such that $H(S)=0$. Land use at $r$ is determined by comparing the wage $w(r)$ with the mixed wage curve $w_{m}(r)$, defined by (8) and (9),

\footnotetext{
${ }^{5}$ In the absence of congestion effects, the existence of equilibrium, and the algorithm's convergence, are proven (see LRH). We assume it also exists in the congested case. The numerical simulations converge, for all the parameter settings that we have experimented with.
} 
respectively. If $w(r)>w_{m}(r)$, the residential bid rent exceeds the business bid rent, so the location is occupied by residents. If $w(r)<w_{m}(r)$, the location is used by firms. Only if $w(r)=w_{m}(r)$, land use may be mixed. According to (9), spatial variation of the mixed wage curve is attributed solely to spatial variation in the productivity function. Therefore, at this step $w_{m}(r)$ is taken as given.

The search for a wage curve proceeds as follows. First, an arbitrary initial wage $w(0)$ at $r_{0}=$ 0 , is chosen. If $w(0)<w_{m}(0)$, then $r_{0}$ is used for business. The innermost zone is then taken by firms, implying inward commuting, and $w(r)$ must be downward sloping. $H(0)=0$, so (8) implies that the slope of $w(0)$ is determined by $k$. As we go farther from the center, $H(r)$ increases, and more units of labor are transported through each location $r$ in order to reach firms at $r_{0}<r$. Congestion levels rise with distance from the center, so $\tau(r)$ increases, and $w(r)$ becomes more concave. This decreasing concave trend of $w(r)$ lasts until we either reach the city border at $r=S$, or $w(r)$ meets $w_{m}(r)$. If $w(r)>w_{m}(r)$, locations further from the center are used by residents only. Then, $H(r)$, which has been increasing in the business zone, is still positive, so commuting occurs inwards. As we now allocate the workers of the business district in their homes, $H(r)$ is decreasing, and so is the congestion level. Therefore, $w(r)$ is convex in this residential region. Finally, when $r=S$, we check whether the land use pattern is consistent, so $H(S)=0$. If $H(S) \neq 0$, the search for an equilibrium starts again using a different guess of $w(0)$.

The opposite occurs if $w(0)>w_{m}(0)$, and the inner city is a residential area. Then, commuting is pointed outwards, and the farther we get from the center, the more residents join the traffic towards the workplaces that may be present in the outer city rings. The rising congestion produces an increasingly convex $w(r)$ curve, until $w(r)$ crosses $w_{m}(r)$.

The picture may be very different if $w(r)$ meets $w_{m}(r)$ when $H(r)=0$, as shown in Figure 1 . A balanced mixed zone may now start at $r$, a zone which exhibits backyard employment, where commuting is absent. In such a zone, $w(r)$ follows the path of $w_{m}(r)$ (independent of the level of transport costs). At any point along this zone, $w(r)$ may depart from $w_{m}(r)$, thus switching to either a residential zone with a wage curve $w_{R}(r)$ lying above $w_{m}(r)$, or to a business zone with a wage curve $w_{B}(r)$ lying below $w_{m}(r)$. 


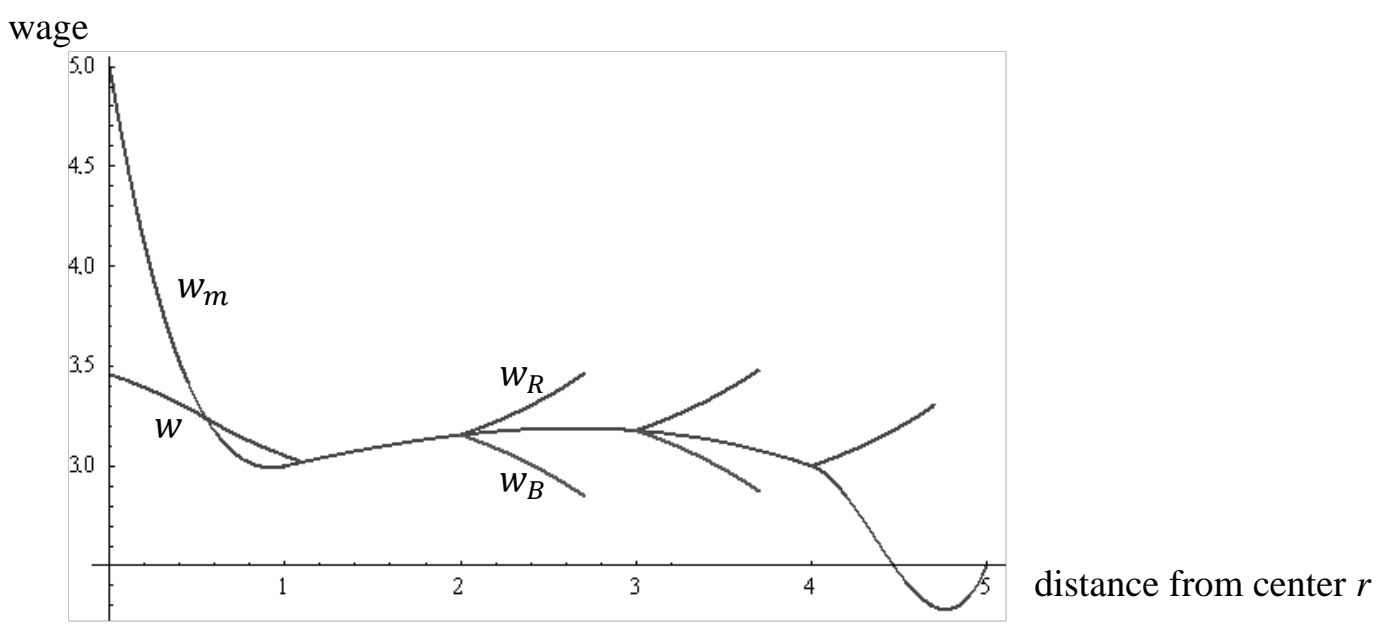

Figure 1: Possible departures from $\mathrm{w}_{\mathrm{m}}$.

Note: A wage function $w$ crosses the mixed wage path $w_{m}$ at $r=1.1$ when $H=0$. It may then overlap $w_{m}$, implying a mixed zone, for as long as the slope of $w_{m}$ does not exceed the marginal commuting costs.

The existence of a mixed zone in equilibrium depends on an important condition: $w_{m}(r)$ must be between $w_{B}(r)$ and $w_{R}(r)$. So, the slope of the wage path following $w_{m}(r)$ must not be larger (in absolute value) than the marginal commuting costs at $r$. If this condition does not hold, a resident might receive a higher wage by changing job location away from the backyard, although the worker incurs positive commuting costs. A firm may similarly offer lower wages to a distant worker, rather than employ an expensive backyard worker. Therefore, when the slope of $w_{m}(r)$ becomes too large, $w(r)$ necessarily departs into a pure zone (business or residential).

The main consequence of the presence of congestion is that $w(r)$ and $w_{m}(r)$ may continue to coincide, even beyond the point where $w_{m}(r)$ becomes too steep (as will be defined below). In order for such a situation to hold in equilibrium, some of the residents of the mixed zone have a positive commute, and congestion occurs. This unbalanced mixed zone may exist if the marginal congested transport costs remain equal to the slope of $w_{m}(r)$.

Consider the following illustration of the emergence of an unbalanced mixed zone, as shown in Figure 2. Suppose the algorithm has constructed land use up to $\tilde{r}$. Suppose that $w(\tilde{r})=w_{m}(\tilde{r})$, 
$H(\tilde{r})=0$, and there is balanced mixed land use at $\tilde{r}^{6}$. Denote a wage path of a pure business zone starting from $\tilde{r}$ by $w_{B}^{\tilde{r}}$. Suppose that $\frac{d w_{m}}{d r}(\tilde{r})=\frac{d w_{B}^{\tilde{r}}}{d r}(\tilde{r})\left(=-\kappa w_{B}^{\tilde{r}}(\tilde{r})\right)$, so $\tilde{r}$ is the point where $w_{m}$ declines too steeply (in the absence of congestion) for a mixed zone to continue to locations to the right of $\tilde{r}$. Commuting from these locations to $\tilde{r}$ provides, then, a higher net wage than working locally, and residents outbid firms for land. In contrast, given congestion, $w$ and $w_{m}$ may continue to coincide, as long as the form of the wage curve is preserved as (8). In this case, we have an unbalanced mixed zone, because $H \neq 0$.

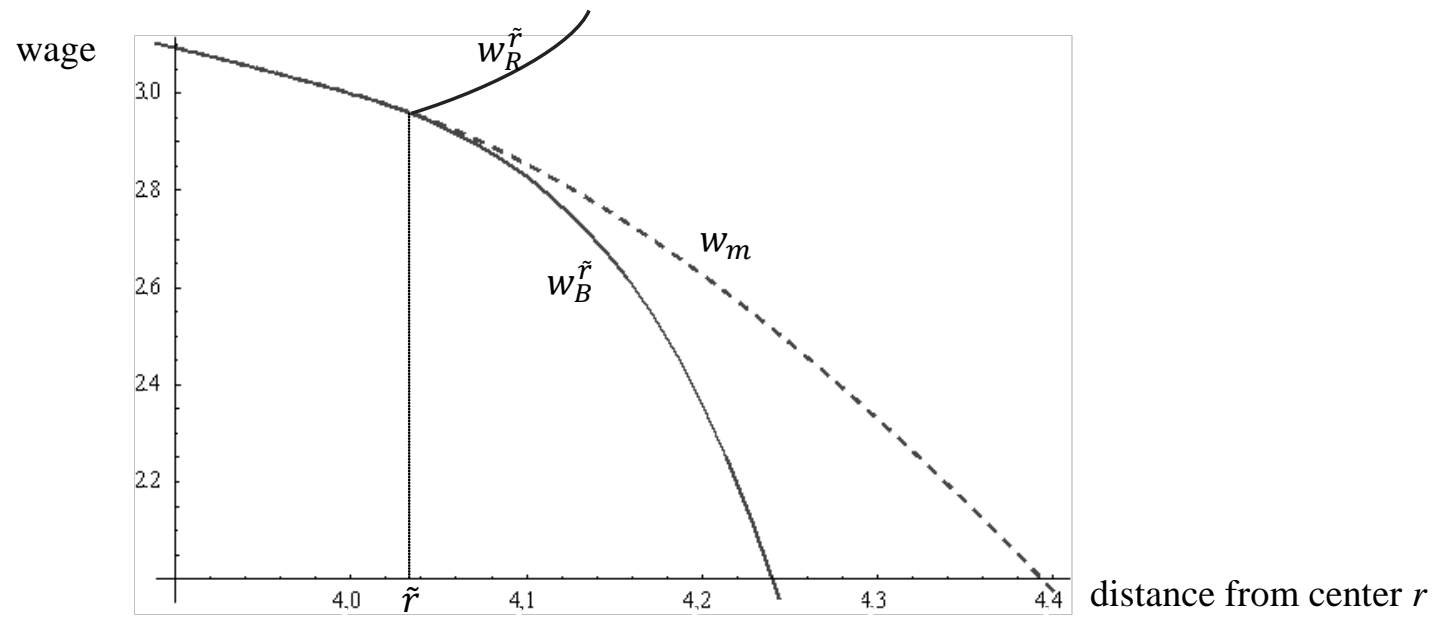

Figure 2: Wage paths at the transition from balanced to unbalanced mixed zone.

Note: With congestion, the wage curve may continue along $w_{m}$, as long as $w_{m}$ is between the wage paths of a pure business zone and a pure residential zone.

The mixed zone may exhibit backyard employment as well as commuting to other zone, as well as within the zone. Since the wage path is downward sloping, commuting is towards the center, and $H(r)$ is positive. The commuters that work in that zone may reside within the zone, as well as in zones further away from the center.

\footnotetext{
${ }^{6}$ An unbalanced mixed zone is not necessarily adjacent to a balanced mixed zone. It may well be the case that $w(r)$ is tangent to $w_{m}(r)$ at a certain location in a pure zone. It may then join the mixed wage path and form an unbalanced mixed zone, as long as (10) holds and $\theta \in(0,1)$.
} 
Let us now examine the necessary conditions for the existence of an unbalanced mixed zone. The wage curve that coincides with $w_{m}$ within the unbalanced mixed zone, is only consistent with (8) given specific values of $\tau$. These $\hat{\tau}$ are given by:

$$
\hat{\tau}(r)=-\frac{1}{w_{m}(r)} \frac{d w_{m}}{d r}(r)
$$

Using this result and (7(9), it follows that:

$$
|H(r)|=2 \pi r\left[\left(\left|-\frac{1}{w_{m}} \frac{d w_{m}}{d r}\right|_{r} \mid-\kappa\right) \frac{1}{t}\right]^{\frac{1}{T}}
$$

where the sign of $H$ is defined by $\operatorname{sgn}(H)=-\operatorname{sgn}\left(\frac{d w_{m}}{d r}\right)$.

Given (11) and (3), the land share occupied by firms, $\theta(r)$, is determined. Since $\theta$ is bounded by the $[0,1]$ interval, the unbalanced mix ed zone can continue as long as $\frac{d H}{d r}(r)$ is smaller (in absolute value) than $\frac{d H}{d r}$ at a pure zone. In other words, an unbalanced mixed location cannot host a larger number of firms than a pure business zone with the same wage and productivity level. Therefore, the necessary conditions for a mixed zone at $\hat{r}$ are $\frac{d w_{m}}{d r}(\hat{r})=-\tau(\hat{r}) w(\hat{r})$ and $\frac{d^{2} w_{R}^{\hat{r}}}{d r^{2}}(r)>$ $\frac{d^{2} w_{m}}{d r^{2}}(r)>\frac{d^{2} w_{B}^{\hat{r}}}{d r^{2}}(r)$, where $w_{R}^{\hat{r}}$ and $w_{B}^{\hat{r}}$ denote wage paths of pure zones starting at $\hat{r}$, in residential and business land uses, respectively.

The second step of the algorithm is described in LRH.

\section{Numerical simulations}

\subsection{The effect of congestion on unbalanced mixed zones}

We will now show the emergence of city structures with unbalanced mixed zones, and explore the mechanism that governs the emergence of such mixed structure, in particular the spatial range of the agglomeration externality, as set by the parameter $\delta$, and the level of congestion determined by $t$ and 
$T$. The algorithm is solved by numerical simulations, in 2000 steps, over a city of 10 -mile radius ${ }^{7}$. We follow LRH in setting the levels of most model parameters ${ }^{8}$, so $f(n)=A n^{\alpha}, g(z)=z^{\gamma}, U(c, l)=$ $c^{\beta} l^{1-\beta}, \gamma=0.04, A=u=1, \alpha=0.95$, and $\beta=0.9$. It is assumed that $k=0.00005$, implying low commuting time costs per mile traveled. This value implies that a traveler loses merely $0.0025 \%$ of their labor time for every one-way mile traveled ${ }^{9}$.

First, we consider a city with $\delta=1$, and without congestion. The agglomeration effect pulls firms to cluster in the center, and the low commuting costs enable residents to travel from distant locations. A monocentric city forms in equilibrium, as shown by LRH. All residents travel through a single zone boundary to get to work. As shown in Figure 3, the density of residents is zero in the inner ring, and the business density is zero in the outer ring.
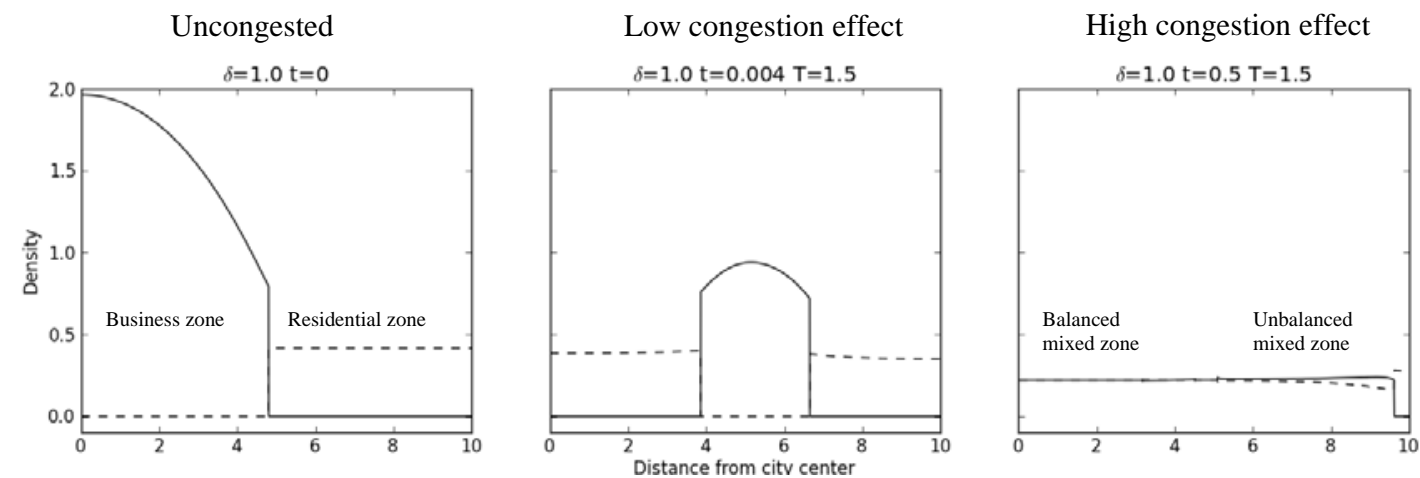

Figure 3: Simulation results of three parameter sets.

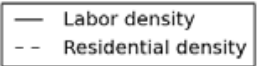

\footnotetext{
${ }^{7}$ The model was solved using the SciPy package. A run to reach equilibrium outcomes such as presented in Figures 3-5 takes about 2 minutes. The code is available on request. Out of the 1000's of city configurations we have obtained, only a few were selected to illustrate the model mechanisms. In discrete simulation problems, numerical accuracy errors arise. To produce smooth city structures, we take advantage of the model property that, given productivity levels, $H(S)$ is a decreasing function of $w\left(r^{\prime}\right)$, for any two wage curves $w_{1}, w_{2}$, where $w_{1}(r)=w_{2}(r)$ for $r<r^{\prime}$. Therefore, we find the closest possible wage curves, one resulting in $H(S)>0$ and the other in $H(S)<0$. Then, we look for $r^{\prime}$, where these two curves diverge from each other, namely where one crosses $w_{m}$. We then find a wage curve starting at r' leading to $\mathrm{H}(\mathrm{S})$ closest to 0 , between the two wage curves.

${ }^{8}$ For a justification of this selection of values, we refer to Rossi-Hansberg (2004).

${ }^{9}$ In all our examples, commuting time is less than $2 \%$ of the production time. Given an eight-hour work day, daily commuting takes 10 minutes, about a quarter of average commuting time in most cities. However, previous studies show that urban models often greatly underestimate total travel time. Hamilton (1982) points to several assumptions of the monocentric model, also made here, which may lead to this discrepancy, including homogeneity and single worker households. LRH assume that total daily travel time is 20 minutes, about half of average time in most empirical studies.
} 
Second, congestion is introduced, where $t=0.004$ and $T=1.5$, so travel time is a convex function of the number of travelers. These values imply that traveling through a road segment used by two units of labor, costs then $1.1 \%=0.004 \cdot 2^{1.5}$ of the remaining labor time per mile traveled. Due to congestion, firms leave the center, and organize in a ring roughly half-way through the city. Agglomeration effects are reduced, and the new firms and residents distribution lowers the travel volumes considerably. The residents of the inner residential zone commute from the center outwards, and the residents of the outer part commute from the outskirts inwards.

Third, we increase the marginal effect of congestion, by setting $t=0.1$. This makes congestion more costly. The structure that emerges exhibits a balanced mixed zone up to 5 miles from the center. It is surrounded by an unbalanced mixed zone, where labor density exceeds residential density. Further out, a narrow pure residential zone completes the city. The workers residing in that zone commute to the unbalanced mixed zone.

The role of the model's key parameters is further illustrated in Figure 4. We consider a case where agglomeration economies are mainly non-local $(\delta=0.4)$, and one where they are mainly local ( $\delta=2.0)$. When commuting is not subject to congestion $(t=0)$, agglomeration benefits at the city center are not offset by high commuting costs. Therefore, the city exhibits a monocentric structure, as shown in panels a and b of Figure 4, and we find that the center has a higher density when agglomeration economies are mainly local.

The city departs from the monocentric structure when we allow for congestion effects $(t>0)$. When $\delta=0.4$, higher levels of congestion effect result in a more complex mixture of land usage (this effect occurs independently of $T$ ). Interestingly, for higher levels of $T$, so the congestion function becomes more convex, unbalanced mixed zones are more common. For example, compare panels e and $\mathrm{k}, \mathrm{h}$ and $\mathrm{n}$, and $\mathrm{m}$ and $\mathrm{s}$.

When $\delta$ is increased to 2, so agglomeration effects are more local, a central location is not necessarily advantageous for firms over a peripheral subcenter. Now, as we increase $T$, the city becomes more dispersed, and subcenters emerge (panels f, l, r and t), spreading employment into mixed zones (compare, for example, $d$ with h, or $\mathrm{j}$ with $\mathrm{n}$ ). 

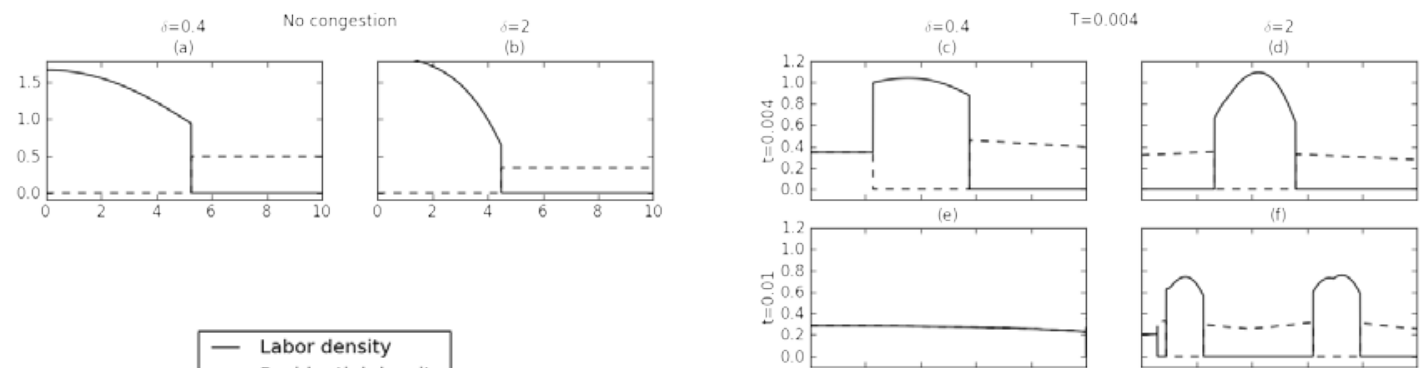

- - Residential density
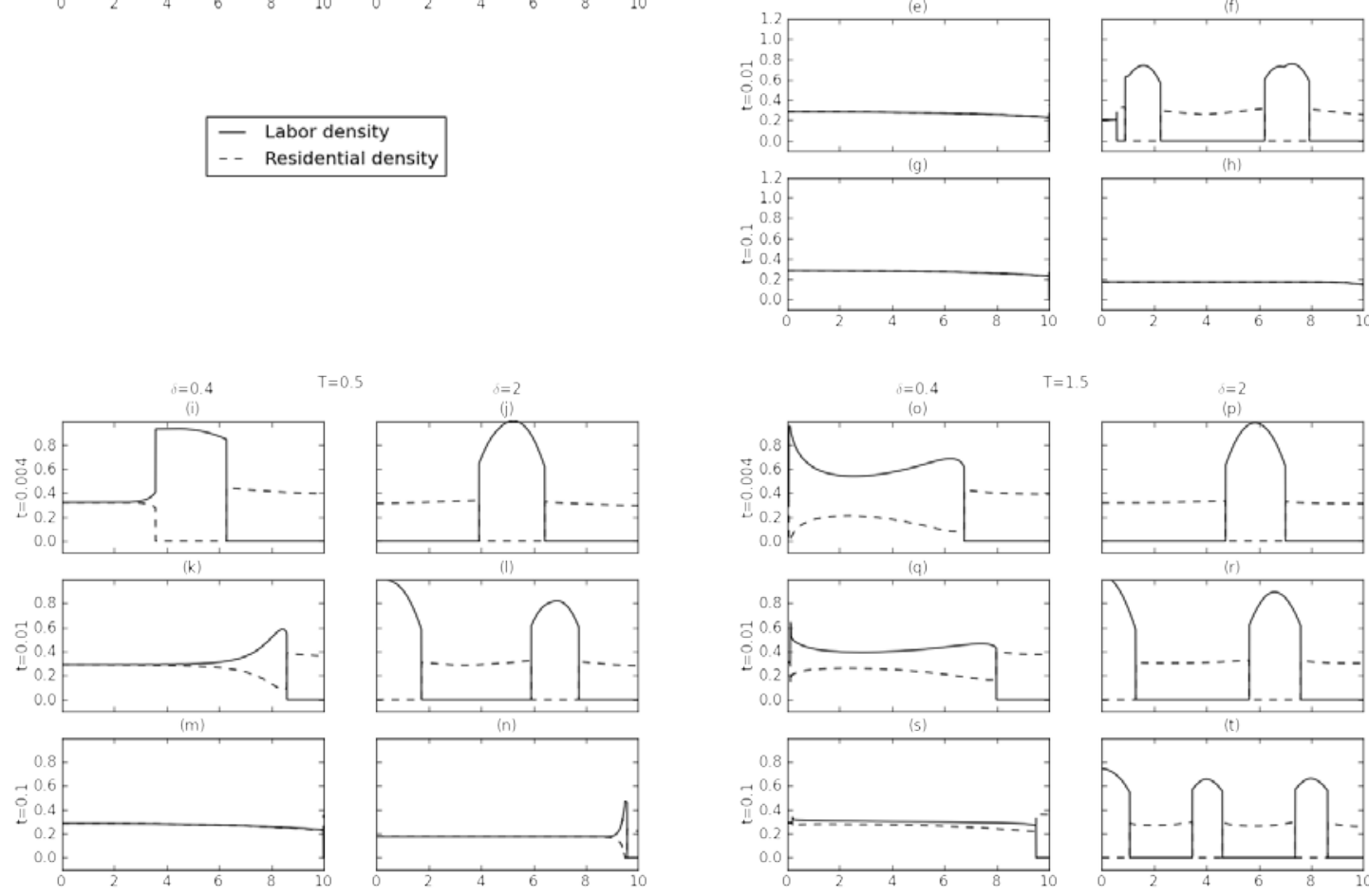

Figure 4: Various congestion and agglomeration settings.

Note: The y-axes indicate densities (of labor and residents), and the x-axes indicate distance from city center.

\subsection{Comparison to Wheaton (2004)}

The urban structures attained by Wheaton (2004) share a remarkably high resemblance to the ones that emerge here ${ }^{10}$. When the congestion function is convex $(T=1.5)$, as assumed in Wheaton, the city exhibits a large business-dominated mixed zone, surrounded by a pure residential ring (Figure 5, panel a). An increase in $t$ can be interpreted as reduced capacity of the road system. As in Wheaton, it results in employment dispersion and a larger central mixed zone (panel b). A large constant transport cost results in a fully mixed, backyard economy city (panel c).

Empirical evidence of land use mixing presented in Wheaton (2004) shows high levels of mixing in two illustrative examples of the American metropolis. Concentration of labor relative to

${ }^{10}$ Compare panels a, b and c of Figure 5 with Figures 6, 10 and 8 in Wheaton (2004). 
residents is higher in New York than in Los Angeles, where the cumulative distributions of labor and residents are nearly identical. Perhaps surprisingly, in the case of New York these distributions are not so far from each other, implying that businesses are not much more concentrated than residents. In our model, this difference can be the result of a higher public transport capacity in New York, captured by a lower $t$. Indeed, as a comparison of panels $\mathrm{o}, \mathrm{q}$ and $\mathrm{s}$ of Figure 4 shows, a higher $t$ leads to a balancing of the distributions of labor and residents. The difference in capacity may be attributed to, for instance, a developed mass transit system in New York, compared with the car-based, congestionprone transportation system of Los Angeles.
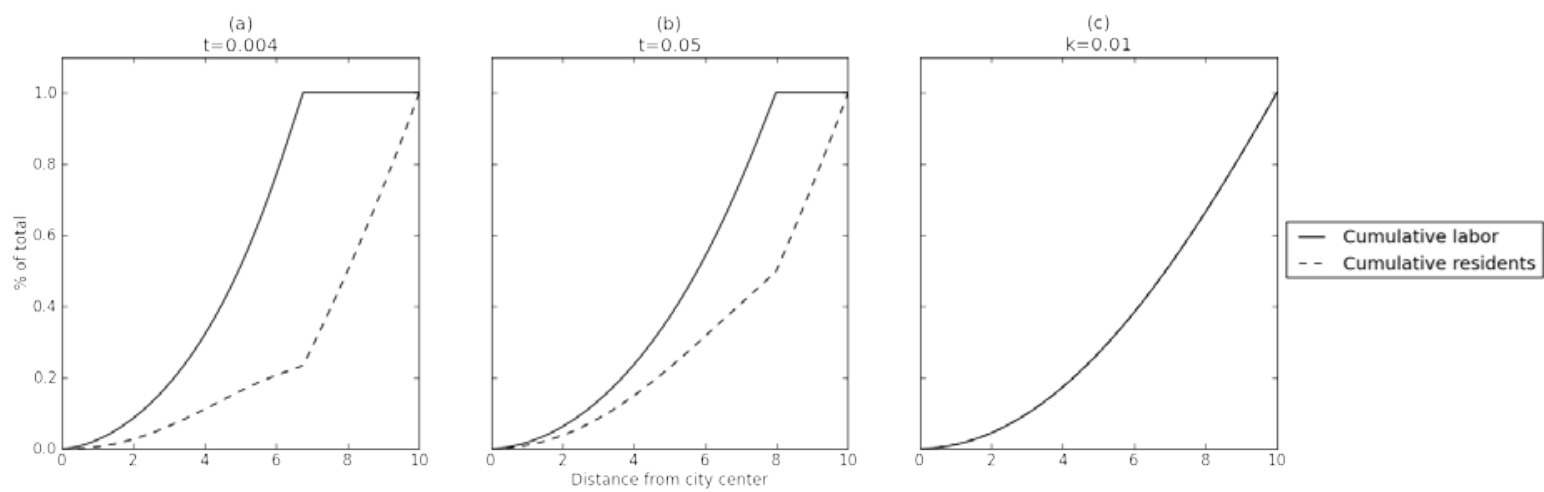

Figure 5: Cumulative employment and residents.

Note: $\delta=0.4, T=1.5$. The panels a and b show results of low and high congestion costs. In panel c, the fixed transport cost is high.

\section{Conclusions}

In this paper we have extended the LRH urban structure model by adding congestion externalities to its transport costs setting. Due to congestion, the model produces an array of urban structures, much wider than the original model can possibly produce. In particular, the land-use map of our model encompasses unbalanced mixed land use zones that contain any arbitrary mix of firms and residents. So, we improve on Wheaton (2004), who essentially assumes mixing. Our theory explains mixed land use, making standard economic assumption of bid-rent competition. We endogenously derive the 
location patterns of households and firms, and the agglomeration externalities they entail, without the need to have recourse to random utility models.

The introduction of a second source of externality has implications for the optimal urban structure as well as for policies aimed at achieving it. This paper has extended the study of LRH, which focused on equilibrium outcomes. The discussion of optimal structure, as done in RossiHansberg (2004) with regards to the LRH model, under the conditions of congestion, is left for future research. We also ignore optimal road policy, which is potentially influenced by the presence agglomeration externalities (see Arnott, 2007).

The sensitive and complex manner, by which the externalities’ parameter settings determine the resulting urban structures, highlights the potential relevance of indirect effects of policy involving any of the externalities. The theory developed illustrates how an improvement in road capacity, reducing congestion, leads to greater concentration of firms, which in turn increases productivity through agglomeration externalities (see panels q and s of Figure 4). This underlines the relevance of indirect benefits of policies to reduce congestion, as also demonstrated by Venables (2007).

\section{References}

Anas, A., R. Arnott and K. A. Small. 1998. “Urban spatial structure”, Journal of Economic Literature, 36:3, pp. 1426-1464.

Anas, A. and I. Kim. "General equilibrium models of polycentric urban land use with endogenous congestion and job agglomeration”, Journal of Urban Economics, 40, pp. 232-256.

Arnott, R. 2007. "Congestion tolling with agglomeration externalities”, Journal of Urban Economics, 62:2, pp. 187-203.

Fujita, M. and H. Ogawa. 1982. "Multiple equilibria and structural transition of non-monocentric urban configurations”, Regional Science and Urban Economics, 12:2, pp. 161-196.

Giuliano, G. and K. A. Small. 1993. “Is the journey to work explained by urban structure?”, Urban Studies, 30:9, pp. 1485-1500.

Glaeser, E. L., H. D. Kallal, J. A. Scheinkman and A. Shleifer. 1992. “Growth in cities”, Journal of Political Economy, 100:6, pp. 1126-1152. 
Hamilton, B. W. and A. Röell. 1982. "Wasteful commuting”, Journal of Political Economy, 90:5, pp. 1035-1053.

Koster, H. R. A. and J. Rouwendal. 2013. “Agglomeration, commuting costs, and the internal structure of cities”, Regional Science and Urban Economics, 43:2, pp. 352-366.

Lucas, R. E. and E. Rossi-Hansberg. 2002. “On the internal structure of cities”, Econometrica, 70:4, pp. 1445-1476.

Mills, E. S. 1972. Studies in the Structure of the Urban Economy. Baltimore: Johns Hopkins Press.

Rosenthal, S. S. and W. C. Strange. 2003. “Geography, industrial organization, and agglomeration”, The Review of Economics and Statistics, 85:2, pp. 377-393.

Rossi-Hansberg, E. 2004. “Optimal urban land use and zoning”, Review of Economic Dynamics, 7:1, pp. 69-106.

Solow, R. 1973. "Congestion costs and the use of land for streets", The Bell Journal of Economics and Management Science, 4:2, pp. 602-618.

Venables, A. J. 2007. "Evaluating urban transport improvements”, Journal of Transport Economics and Policy, 41:2, pp. 173-188.

Wheaton, W. C. 2004. “Commuting, congestion, and employment dispersal in cities with mixed land use”, Journal of Urban Economics, 55:3, pp. 417-438. 